\title{
Evidence for acupoint catgut embedding treatment and TRPV1 gene deletion increasing weight control in murine model
}

\author{
CHANYA INPRASIT ${ }^{1}$, YU-CHUEN HUANG ${ }^{2,3}$ and YI-WEN LIN ${ }^{1}$ \\ ${ }^{1}$ College of Chinese Medicine, Graduate Institute of Acupuncture Science, China Medical University; \\ ${ }^{2}$ College of Chinese Medicine, School of Chinese Medicine, China Medical University; ${ }^{3}$ Department of \\ Medical Research, China Medical University Hospital, Taichung 40402, Taiwan, R.O.C.
}

Received August 23, 2019; Accepted December 12, 2019

DOI: $10.3892 /$ ijmm.2020.4462

\begin{abstract}
Obesity is a global health problem affecting the general population. Acupoint catgut embedding (ACE) is an alternative treatment that involves the implantation of absorbable catgut suture at acupoints. The transient receptor vanilloid member 1 (TRPV1) is a calcium ion channel that responds to several chemical ligands and is identified in numerous locations throughout the body. The aim of the present study was to examine the effect of ACE treatment on obesity and its associated complications through various neural mechanisms in a murine model. A C57/BL6 wild type (WT) and TRPV1 ${ }^{-/}(\mathrm{KO})$ mouse model was utilized to exclude any psychological factors associated with obesity. The WT-HFD-ACE and WT-HFD-SHAM groups received weekly ACE or placebo treatments at the bilateral ST36 acupoint. The mice were fed with a normal mice chow diet (ND) or a high-fat food diet (HFD; $45 \mathrm{kcal} \%$ ), and their body weights were recorded once a week. After 8 weeks, the subjects were sacrificed and changes in the levels of a number of biomarkers were investigated using ELISA, immunoblotting and immunofluorescence. The results indicated a significant decrease in
\end{abstract}

Correspondence to: Professor Yi-Wen Lin, College of Chinese Medicine, Graduate Institute of Acupuncture Science, China Medical University, 91 Hsueh-Shih Road, Taichung 40402, Taiwan, R.O.C.

E-mail: yiwenlin@mail.cmu.edu.tw

Professor Yu-Chuen Huang, College of Chinese Medicine, School of Chinese Medicine, China Medical University, 91 Hsueh-Shih Road, Taichung 40402, Taiwan, R.O.C.

E-mail: yuchuen@mail.cmu.edu.tw

Abbreviations: ACE, acupoint catgut embedding; ST36, Zusanli acupoint; NTS, nucleus tractus solitarii; PFC, prefrontal cortex; sWAT, subcutaneous white adipose tissue; vWAT, visceral white adipose tissue; BAT, brown adipose tissue; WT, wild type mouse; KO, TRPV1 knockout mouse; ND, normal diet; HFD, high fat diet

Key words: acupoint catgut embedding, obesity, hypothalamus transient receptor potential vanilloid 1 , prefrontal cortex body weight variation for the WT-HFD-ACE group compared with the WT-HFD and WT-HFD-SHAM groups, using the WT-ND group as the body weight baseline. By contrast, KO mice fed with ND or HFD demonstrated notable body weight maintenance throughout the experimental period. Similar patterns were observed in adipose tissue mass, glucose, leptin and insulin plasma levels, and protein molecule density of TRPV1 and its associated molecules in the hypothalamus and nucleus tractus solitarii. In contrast, in the prefrontal cortex, significant decreases in the concentrations of MAPK pathway proteins in the WT-HFD and WT-HFD-SHAM groups were observed. The levels of these proteins were significantly increased in the WT-HFD-ACE and KO-HFD groups. These results suggested that TRPV1 and its associated pathways may be involved in body weight maintenance, and may be controlled through ACE treatment or genetic manipulation.

\section{Introduction}

Obesity is a chronic non-communicable disease, the rate of which has been rising exponentially on a global scale (1). It is ranked among the top 10 risk factors associated with several other conditions, such as type II diabetes mellitus, dyslipidemia and cardiovascular disease (2). Furthermore, obesity increases the incidence of psychological diseases, in particular major depressive disorder (3). Several neural mechanisms associated with obesity may occur in the hypothalamus and nucleus tractus solitarii (NTS). The hypothalamus and NTS are areas of the brain that are primarily concerned with food intake and energy expenditures $(4,5)$. Food intake responses, including appetite and satiety senses, are regulated through the release of hormones and molecules from the stomach, adipose tissue, the intestines, the pancreas, the pituitary gland, the hypothalamus and the NTS. The functions of this pathway are relatively clear when observed in isolation. After the central nervous system receives the relevant signals from organs, it secretes orexigenic and anorexigenic peptides responsible for the amount of food consumed $(6,7)$. Pathologically, the etiology of obesity is attributed to the total number and size of adipocytes, which include the cells that make up white adipose tissue (WAT) and brown adipose tissue (BAT). Previous studies have identified that BAT is able to produce heat and energy. This mechanism increases energy expenditures, thermogenesis, and 
the oxidation of lipids and may influence the development of obesity $(8,9)$.

The transient receptor potential vanilloid 1 (TRPV1) is one of 6 members of the cation channel subfamily. TRPV1 is the first unit in this sequence to be specified and most keenly characterized, due to its ability to act as a homotetrameric, nonselective, calcium-permeable cation channel $(10,11)$. TRPV1 is well known for its role in nociception and inflammation, which may occur at lowered $\mathrm{pH}$ levels $(\mathrm{pH}$ 6.0) and high temperatures $\left(43^{\circ} \mathrm{C}\right)(12)$. Aside from the factors already mentioned, there are other factors that are associated with TRPV1 function, such as pathological weight gain and certain psychosomatic disorders $(13,14)$. This response causes a signaling cascade in neurons via the activation of protein kinases, including mitogen-activated protein kinase (MAPK), and cyclic AMP-response element binding protein (CREB) (15). These protein kinase families are the secondary messengers that mediate intracellular signaling. These kinases include protein kinase A (PKA) and protein kinase C (PKC). These signaling cascades are important for maintaining homeostasis and may be stimulated and/or inhibited by various factors such as acupuncture. A number of pathological mechanisms are associated with the dysregulation of one, many, or all of the factors associated with these signaling pathways $(16,17)$.

The theory of Traditional Chinese Medicine introduced the concept of obesity as a result of dysfunction within the gastrointestinal organs, primarily the spleen and the stomach $(18,19)$. Acupuncture has been used as a traditional method for treating several diseases, including weight control. The combination of acupuncture and lifestyle modifications may significantly improve weight loss (20). Acupoint catgut embedding (ACE) involves the implantation of absorbable catgut sutures at traditional Chinese meridian acupoints. This technique is believed to confer beneficial therapeutic effects: Notable decreases in treatment frequency from approximately twice a week to twice a month have been observed (21). The ability to conserve time and costs makes ACE an attractive treatment method. Although side effects such as local inflammation, pain, callosity, and discomfort may occur for several days following treatment, this method has been widely used for the management of obesity (22). Zusanli (ST36) is most commonly used for the treatment of pain, lower limb issues and gastrointestinal disorders. In TCM, it is believed that it may also be used to treat diseases that manifest along the stomach meridian. In addition to the positive effects associated with using ACE to treat obesity, ST36 is also frequently combined with local abdominal acupoints to treat obesity and maintain normal body weight (23). The purpose of the present study was to investigate the effect of ACE on obesity and its associated complications by identifying various neural mechanisms associated with the disease. Therefore, we hypothesized that ACE treatment may decrease the incidence of obesity through the control and maintenance of body weight. In addition, we also hypothesized that ACE treatment would also decrease the complications associated with obesity via modulation of TRPV1 signaling.

\section{Materials and methods}

Experimental animals. A total of 28 female C57/BL6 wild type (WT) mice and 14 female TRPV1 knockout (KO) mice that were 7 weeks old and weighing 19-22 g, were purchased from BioLASCO Taiwan Co., Ltd. The animals were housed individually in cages under a 12:12 h light-dark cycle (from 8.00 a.m. to 8.00 p.m.), at a temperature of $25 \pm 1^{\circ} \mathrm{C}$, and humidity of $60 \pm 5 \%$ relative humidity, with ad libitum access to water and a normal mice chow diet (ND) for 1 week prior to initiation of the experiment. The study was approved by the Institute of Animal Care and USE Committee of China Medical University (Permit no. 2016-061), following the Guide for the Care and Use of Laboratory Animals (24). The first day of experiment, the cages, animal bedding and water were changed, and the mice chow was superseded by high-fat diet (HFD) (Research Diets Inc.; cat. no. D12451) composed of $45 \mathrm{kcal} \%$ fat (1,598 kcal\% of lard; $225 \mathrm{kcal} \%$ soybean oil, USP), $35 \mathrm{kcal} \%$ carbohydrate and $20 \mathrm{kcal} \%$ protein $(473 \mathrm{kcal} / 100 \mathrm{~g})$. Subjects were randomly divided into six groups: Control group (WT-ND); obesity group (WT-HFD); Acupoint catgut embedding (WT-HFD-ACE) group; sham acupoint catgut embedding (WT-HFD-SHAM) group; TRPV1 knockout mice with normal diet (KO-ND); and TRPV1 knockout mice with HFD (KO-HFD), with 7 mice in each group. All food of the subjects was weighed before being replenished with either ND or HFD mice chow weekly. The subjects were weighed once a week throughout the 8 week experimental period to study weight changes. Food was collected, measured, refilled and replaced to detect food consumption patterns on the same day following body weight measurement. The cages were cleaned once a week, with the animal bedding and water also changed. A preliminary pilot study was conducted with 4 animals to analyze the absorbable ability of catgut at various time intervals. All 4 subjects were treated via insertion of absorbable catgut into the ST36 acupoint. After 7 days, the implantation area of 2 mice was incised, and the implantation area of the remaining 2 mice was incised at 14 days after the embedding to establish the ideal absorbability duration. The experiment was designed to sacrifice that the mice at the end of the 8th week of the experiment. They were fasted with no access to food but were fed water ad libitum $12 \mathrm{~h}$ prior to sacrifice. Efforts were made to minimize the number of animals used and their suffering. A total of 8 subjects were removed during the experimental period due to excessive food gnawing behavior and weight gain or loss at $25 \pm 1 \mathrm{~g}$, as established in Week 4 prior to initiation of the treatment session.

Acupoint catgut embedding treatment. Mice in the WT-HFD-ACE group received ACE treatment at the bilateral side of ST36 once a week on the first day of the 5 to 8 th week. In mice, as in humans, the ST36 point is located longitudinally at 3 cun below the knee joint and intersects with the middle of the tibialis anterior muscle (25). Sterile conventional syringe needles $0.6 \times 25 \mathrm{~mm}$ (Terumo Corporation), acupuncture needles $0.35 \times 40 \mathrm{~mm}$ (Suzhou Medical Appliance) and brown catgut $0.2 \times 4 \mathrm{~mm}$ (CP Medical Inc.) were used for the implantation. Animals from the WT-ND, WT-HFD, KO-ND and KO-HFD groups were placed into a fixation machine under anesthesia with 5\% isoflurane for induction, which was then decreased to $1 \%$ for maintenance. Bilateral ST36 acupoint were selected, sterilized with $70 \%$ alcohol and iodine solution and the catgut was embedded at the $5 \mathrm{~mm}$ depth before the animals were returned to individual observation home cages 
after the procedure. The subjects in the WT-HFD-SHAM group received sham ACE treatment, which consisted of using similar equipment to insert the empty needle at the ST36 acupoint without catgut implantation. The needle embedding causes a minute red mark at the area of needle insertion, which was fully recovered within $48 \mathrm{~h}$ after the treatment. In addition, there was no apparent difficulty of movement following anesthesia.

Collection of samples. In the samples collection process, after subjects were fasted for $12 \mathrm{~h}, 38$ subjects were euthanized with $5 \%$ isoflurane by inhalation. Blood samples were collected from the Orbital sinus into $3 \mathrm{ml} \mathrm{BD} \mathrm{Vacutainer} \mathrm{glass}$ tubes with $5.4 \mathrm{mg} \mathrm{K} 2$ EDTA and $2 \mathrm{ml}$ BD Vacutainer glass tubes with $3 \mathrm{mg}$ sodium fluoride and $6 \mathrm{mg} \mathrm{Na} \mathrm{ND}_{2}$ EDA. The samples were centrifuged at $1,500 \mathrm{x}$ g for $15 \mathrm{~min}$ at $25^{\circ} \mathrm{C}$, following which the separated plasma was collected into $1.5 \mathrm{ml}$ microcentrifuge tubes and stored at $-80^{\circ} \mathrm{C}$. Following collection of the blood samples, the animals were decapitated, and brains were excised for western blot analysis. The adipose tissues were then collected from various regions of the body; BAT from the interscapular area, subcutaneous WAT (sWAT) from both sides of the flank area and visceral WAT (vWAT) from the perigonadal area (26). Finally, the liver and both kidneys were immediately dissected out and weighed.

Western blot analysis. Western blot analysis is an analytical technique that is widely used to study proteins. This method, first described by Towbin et al (27), uses an antibody that recognizes and binds to an epitope unique to the protein of interest. In the present study, following sacrifice, the hypothalamus, prefrontal cortex (PFC) and NTS were immediately dissected out and frozen in ice prior to storage at $-80^{\circ} \mathrm{C}$. Total proteins were prepared by abrasion and lysis in solution of $50 \mathrm{mM}$ Tris- $\mathrm{HCl}$ pH 7.4, $250 \mathrm{mM} \mathrm{NaCl}, 1 \%$ NP-40, 5 mM EDTA, $50 \mathrm{mM} \mathrm{NaF}$, $1 \mathrm{mM} \mathrm{Na} \mathrm{VO}_{4}, 0.02 \% \mathrm{NaN}_{3}$ and $1 \mathrm{X}$ protease inhibitor cocktail (Amresco, LLC) prior to centrifugation at $10,000 \mathrm{x} \mathrm{g}$ for $10 \mathrm{~min}$ at $4^{\circ} \mathrm{C}$. Proteins from each sample were loaded into $8 \%$ SDS-Tris glycine gel electrophoresis gels and transferred onto PVDF membranes, which were then blocked with 5\% non-fat milk in TBS-T buffer (10 mM Tris pH 7.5, $100 \mathrm{mM} \mathrm{NaCl}, 0.1 \%$ Tween-20), and incubated for $1 \mathrm{~h}$ at room temperature with the following primary antibodies: Anti-TRPV1 ( 95 kDa; Alomone; cat. no. ACC-030; 1:1,000), anti-p-PI3K ( 110 kDa; Novus Biologicals, LLC; cat. no. NBP2-15071; 1:1,000), anti-PI3K ( 126 kDa; Abcam; cat. no. ab154598; 1:1,000), anti-phosphorylated (p)-Akt $(\sim 65 \mathrm{kDa}$, Thermo Fisher Scientific, Inc.; cat. no. 44-621G; 1:1,000), anti-Akt ( 65 kDa; Merck KGaA; cat. no. 16-293; 1:1,000), anti-p-mTOR ( 289 kDa; Merck KGaA; cat. no. 09-213; 1:1,000), anti-mTOR ( 238 kDa; Abcam; cat. no. ab2732; 1:1,000), anti-p-extracellular signal-regulated kinase (ERK) 1/2 (p-ERK) 42 kDa; Abcam; cat. no. ab138482; 1:1,000), anti-ERK ( 42-44 kDa; Cell Signaling Technology, Inc.; cat. no. 4695; 1:1,000), anti-p-c-Jun N-terminal kinase (p-JNK; 45-55 kDa; Thermo Fisher Scientific, Inc.; cat. no. 44-682G; 1:1,000), anti-JNK ( 46-54 kDa; Cell Signaling Technology, Inc.; cat. no. 9252; 1:1,000), anti-p-p38 mitogen-activated protein kinase (p-p38; $\sim 41 \mathrm{kDa}$; Thermo Fisher Scientific, Inc.; cat. no. 44-684G; 1:1,000), anti-p38 ( 43 kDa; Cell Signaling Technology,
Inc.; cat. no. 9212; 1:1,000), anti-p-NF- $\mathrm{B}(\sim 65 \mathrm{kDa}$; Merck

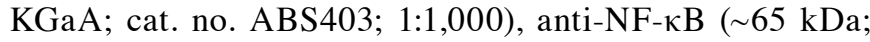
Cell Signaling Technology, Inc.; cat. no. 8242; 1:1,000), anti-p-CREB ( 43 kDa; Merck KGaA; cat. no. 06-519; 1:1,000), anti-CREB ( 43 kDa; Cell Signaling Technology, Inc.; cat. no. 9197; 1:1,000), anti-p-protein kinase $C$ epsilon type (p-PKC $\varepsilon ; ~ 82 \mathrm{kDa}$; Santa Cruz Biotechnology, Inc.; cat. no. SC-12355; 1:1,000), anti-PKCe ( 84 kDa; Abcam; cat. no. ab63638; 1:1,000), anti-p-protein kinase AII $\alpha$ (p-PKAII $\alpha$; 40 kDa; Santa Cruz Biotechnology, Inc.; cat. no. SC-12905; 1:1,000) or anti-PKAII $\alpha(\sim 40$ kDa; Santa Cruz Biotechnology, Inc.; cat. no. SC-136262; 1:1,000) in TBST with $1 \%$ bovine serum albumin (BSA) (Sigma-Aldrich; Merck $\mathrm{KGaA}$ ). Horseradish peroxidase-conjugated AffiniPure goat anti-mouse (Jackson ImmunoResearch Laboratory, Inc.; cat. no. 115-035-003; 1:5,000), goat anti-rabbit (Jackson ImmunoResearch Laboratory, Inc; cat. no. 111-035-003; 1:5,000) and donkey anti-goat (Jackson ImmunoResearch Laboratory, Inc.; cat. no. 705-035-003; 1:5,000) secondary antibodies were incubated with the membranes for $1 \mathrm{~h}$ incubation at room temperature. The protein bands on the membranes were visualized using an enhanced chemiluminescent substrate kit (Pierce; Thermo Fisher Scientific, Inc.) with LAS-3000 Fujifilm (Fuji Photo Film Co. Ltd). The image densities of the specific bands were quantified by National Institutes of Health (NIH) ImageJ software (version 1.8.0).

Immunofluorescence. A total of 4 subjects, from the WT-HFD, WT-HFD-ACE, WT-HFD-SHAM, and KO-HFD groups, were anesthetized using $1 \%$ isoflurane by inhalation and intracardially perfused with saline followed by $4 \%$ paraformaldehyde. The brain was immediately dissected and post-fixed with $4 \%$ paraformaldehyde at $4{ }^{\circ} \mathrm{C}$ overnight. Post-fixed tissues were placed overnight in $30 \%$ sucrose for cryoprotection at $4^{\circ} \mathrm{C}$. The brain was embedded in OCT and instantaneously frozen using liquid nitrogen prior to storage at $-80^{\circ} \mathrm{C}$. Frozen segments were cut at a thickness of $16 \mu \mathrm{m}$ width on a cryostat then placed on glass slides. The samples were incubated with blocking solution, which consisted of 3\% BSA, $0.1 \%$ Triton $\mathrm{X}-100$ and $0.02 \%$ sodium azide, for $2 \mathrm{~h}$ at room temperature. Following blocking, the brain samples were incubated overnight with the primary antibody, TRPV1 ( 95 kDa; Alomone; cat. no. ACC-030; 1:200), pPKAII $\alpha(\sim 40$ kDa; Santa Cruz Biotechnology, Inc.; cat. no. SC-12905; 1:200), p-PI3K ( $110 \mathrm{kDa}$; Novus Biologicals, LLC; cat. no. NBP2-15071; 1:200), and p-CREB ( 43 kDa; Merck KGaA; cat. no. 06-519; 1:200), prepared in BSA solution at $4^{\circ} \mathrm{C}$ overnight. The secondary antibodies, Alexa Fluor 488-conjugated AffiniPure donkey anti-rabbit (Jackson ImmunoResearch Laboratory, Inc.; cat. no. 711-545-152; 1:500), donkey anti-mouse (Jackson ImmunoResearch Laboratory, Inc., cat. no. 715-545-150; 1:500) and 594-conjugated AffiniPure donkey anti-goat (Jackson ImmunoResearch Laboratory, Inc.; cat. no. 705-585-003; 1:500) were used for incubation at room temperature for $2 \mathrm{~h}$ prior to being fixed with cover slips for immunofluorescence visualization. The samples were observed by an epi-fluorescent microscope (BX-51; Olympus Corporation) with x 200 total magnification (x20 numerical aperture $(\mathrm{NA}=0.4)$ objective lens and x10 ocular lens). The images were analyzed by NIH ImageJ software (version 1.8.0). 
ELISA. The level of mouse leptin (cat. no. KMC2281) and insulin (cat. no. EMINS) in the blood plasma were detected using ELISA kits following the manufacturer's protocol (Thermo Fisher Scientific, Inc.; catalog no. EIAGLUC). The ELISA procedures were prepared at room temperature. The plasma samples were placed in 96-well plates with mouse leptin or insulin antibody coating prior to incubation at $25^{\circ} \mathrm{C}$ for $2 \mathrm{~h}$. They were then removed from the plate and washed with wash buffer. The biotinylated antibody reagent was added in the wells and incubated for $1 \mathrm{~h}$ before the washing process. The wells were then incubated at $25^{\circ} \mathrm{C}$ for $30 \mathrm{~min}$ with a streptavidin-HRP reagent and then washed. Next, tetramethylbenzidine substrate was added into each well to incubate for $30 \mathrm{~min}$ in darkness. Following the addition of the stop solution, the absorbent substance at $450 \mathrm{~nm}$ was measured using a microplate reader. The glucose level was measured with the glucose colorimetric detection kit (Thermo Fisher Scientific, Inc., EIAGLUC); in this procedure, the principles of the glucose oxidation reaction with glucose to produce hydrogen peroxide were used; the resulting colored product was then read by the microplate reader at $450 \mathrm{~nm}$ for leptin plasma level and $550 \mathrm{~nm}$ for insulin and glucose plasma levels. Finally, the samples were measured and analyzed using the kit-specific standard CurveExpert Basic (version 2.1.0; Hyams Development).

Statistical analysis. Statistical analysis was performed using the SPSS statistical software package program v.22.0 (IBM Corp.). All data are presented as the mean \pm standard error of the mean. Statistical comparisons were evaluated using a one-way analysis of variance followed by Tukey's post hoc test to determine the significance of differences. $\mathrm{P}<0.05$ was considered to indicate a statistically significant difference.

\section{Results}

Effect of ACE treatment on weight control. The simple standard of obesity in the mouse model was indicated by an increase in net body weight throughout the 8 -week experimental period. All analyzed data are presented in Fig. 1A. During this experiment, a significant difference in weight gain between the WT and the TRPV1 KO groups was identified. This was observed in the initial increase in body weight observed in the WT-ND, KO-ND, and KO-HFD groups compared with that in the WT-HFD, WT-HFD-ACE, and WT-HFD-SHAM groups at Week 4. A significant increase in body weight was observed in the WT groups, with the WT-HFD-ACE group demonstrating a gradual decrease in Week 7 and significant difference in Week 8. At Week 8, there was a slight decrease in body weight observed in the WT-HFD-SHAM group. Although the mean body weight of the WT-HFD-SHAM group was decreased compared with that of the WT-HFD group, a significant difference in body weight between the WT-HFD-ACE and WT-HFD-SHAM groups was observed. At Week 4, there was a significant decrease in the KO-ND group compared with that in the WT-ND group. Concomitantly, no significant difference between the WT-ND and KO-HFD groups was observed at this time. Food intake among the groups was also examined. A significant increase in food consumption was observed in the WT-ND and KO-ND groups from the first week of the experiment compared with that of the other groups. Furthermore, a significantly decreased level of food consumption was observed in the KO-HFD group compared with that in the other HFD-fed groups (Fig. 1B). The results demonstrated a slight decrease in food intake in the WT-HFD-ACE group following ACE treatment compared with that in the WT-HFD and WT-HFD-SHAM groups. However, food consumption in the WT-HFD-ACE group significantly decreased during the 8th week compared with that in the WT-HFD group. A nonsignificant increase in food consumption across all groups was observed in accordance with the growth requirements of the mice.

Effect of ACE treatment on tissue weight. The tissue weights, including the weights of adipose tissue and associated organs, were measured and recorded immediately following euthanasia. Statistically significant differences in the weight of adipose tissue were consistent with differences in net body weight. Compared with those in the ND-fed groups, significant increases in the total recorded BAT, sWAT, and vWAT weights were observed in the WT-HFD-fed groups (Fig. 2B-D). The data revealed significant decreases in the weights of BAT and vWAT in the WT-HFD-ACE groups compared with those in the WT-HFD group. Furthermore, there was significant decrease in liver weight in the WT-ND, the TRPV1 KO and both the ND and HFD groups when compared with that of the other three WT groups. Conversely, there were significant decreases in net kidney weight observed in the KO-ND and KO-HFD groups compared with that in the WT-ND, WT-HFD, WT-HFD-ACE, and WT-HFD-SHAM groups. No statistically significant differences were observed among the four WT groups.

Glucose, insulin and leptin plasma level changes following a $12 \mathrm{~h}$ fast. At $12 \mathrm{~h}$, there was a significant difference in fasting plasma glucose levels in the WT-HFD and WT-HFD-SHAM groups, which were induced to become obese without any treatment interventions. However, plasma glucose levels were significantly diminished in the WT-HFD-ACE group following the receipt of therapy, and in the KO-HFD group. Analysis of the insulin plasma concentrations in the four groups revealed that the levels of this hormone were significantly decreased in the WT-HFD-ACE group compared with those in the WT-HFD and WT-HFD-SHAM groups. There was also a significant decrease in insulin plasma levels in the KO-HFD group compared with that in the other groups. Additionally, the levels of leptin, which is a hormone released by adipocytes to produce feelings of satiety, exhibited the same pattern to that observed in the insulin plasma graph. The changes in leptin plasma levels within the WT-HFD, WT-HFD-ACE, WT-HFD-SHAM, and KO-HFD groups are presented in Fig. 3C.

Effect of ACE treatment on proteins of TRPVI and associated molecules in the hypothalamus. Western blot analysis was used to determine the effect of ACE treatment on the expression levels of the TRPV1 receptor and associated downstream molecules. TRPV1 protein levels significantly decreased following the receipt of treatment. This decrease was observed in the WT-HFD-ACE and KO-HFD groups. A significant 
A
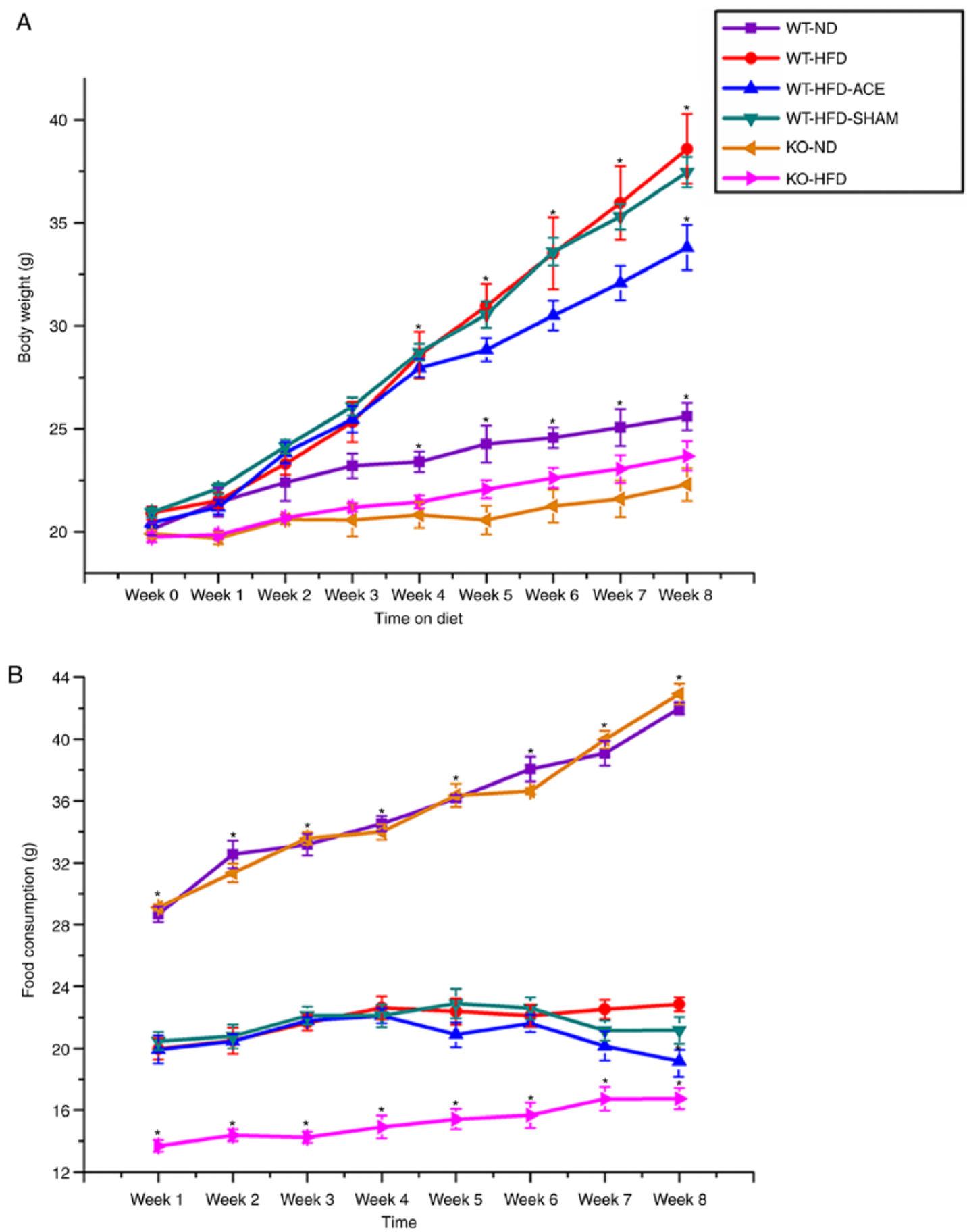

Figure 1. Weekly body weight alterations and food consumption in the six subject groups. (A) The graph presents comparisons of body weight in the WT-ND, WT-HFD, WT-HFD-ACE, WT-HFD-SHAM, KO-ND and KO-HFD groups. Significant body weight increases in the WT-HFD, WT-HFD-ACE and WT-HFD-SHAM groups compared to the WT-ND group and both TRPV1 KO mouse groups were observed. " $\mathrm{P}<0.05$. There was significant body weight decease in the WT-HFD-ACE group compared to the WT-HFD group and WT-HFD-SHAM group. ${ }^{*} \mathrm{P}<0.05$. (B) The graph presents comparisons in food consumption in the WT-ND, WT-HFD, WT-HFD-ACE, WT-HFD-SHAM, KO-ND and KO-HFD groups. Significant increases of food intake in all normal mice chow, WT-ND and KO-ND groups compared to the HFD-fed mouse groups were observed. "P<0.05. A significant difference in food consumption was also observed for the KO-HFD group when compared with the WT-HFD, WT-HFD-ACE and WT-HFD-SHAM groups. There was significant decrease of food intake in the WT-HFD-ACE group compared with the WT-HFD group in Week 8. "P<0.05. WT, wild-type; ND, normal diet; HFD, high-fat diet; ACE, acupoint catgut embedding; KO, knockout.

increase in TRPV1 expression was observed in the WT-HFD and WT-HFD-SHAM treatment groups, which indicated increased amounts of adipose tissue storage, and hence weight gain. The levels of p-PI3K, p-Akt and p-mTOR were significantly increased in the WT-HFD and WT-HFD-SHAM groups. However, the levels of these phosphorylated proteins were significantly decreased in the WT-HFD-ACE and KO-HFD groups. Similarly, a significant decrease in levels of phosphorylated proteins from the MAPK pathway, specifically p-ERK, pp38 and p-JNK, was observed. There were also significant decreases in levels of p-PKC $\varepsilon$ and p-PKAII $\alpha$ in the WT-HFD-ACE and KO-HFD groups when compared to those 
A
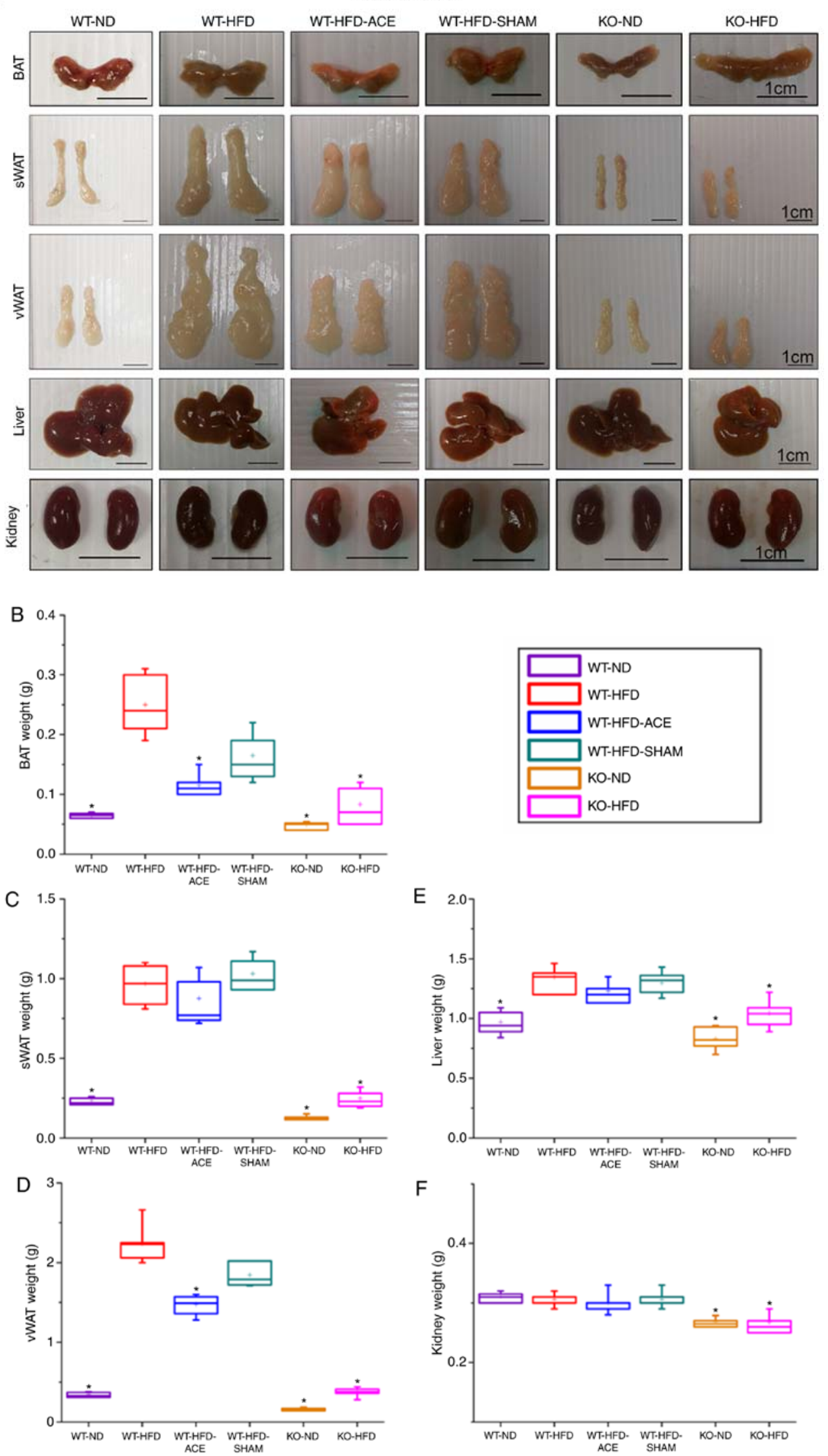

Figure 2. BAT, sWAT, vWAT, liver and kidney weight and dimension in the six subject groups. The comparisons in (A) tissue dimension and (B-F) tissue weight in the WT-ND, WT-HFD, WT-HFD-ACE, WT-HFD-SHAM, KO-ND and KO-HFD groups. Significantly increased BAT and vWAT levels were observed in the WT-HFD and WT-HFD-SHAM groups compared with the WT-ND, WT-HFD-ACE, KO-ND and KO-HFD groups, which exhibited significantly smaller values. The sWAT and liver results presented significant decreases in the WT-ND, KO-ND, and KO-HFD groups, whilst only a significant decrease in kidney weight was observed in two KO groups. "P<0.05. BAT, brown adipose tissue; sWAT, subcutaneous white adipose tissue; vWAT, visceral white adipose tissue; WT, wild-type; ND, normal diet; HFD, high-fat diet; ACE, acupoint catgut embedding; KO, knockout. 
A

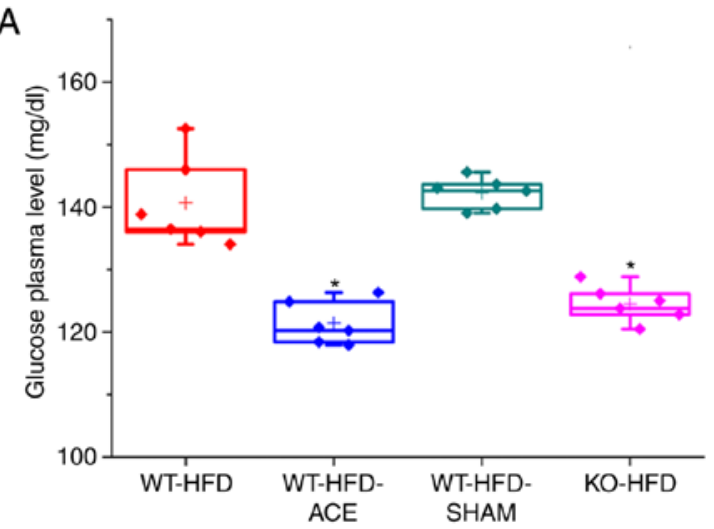

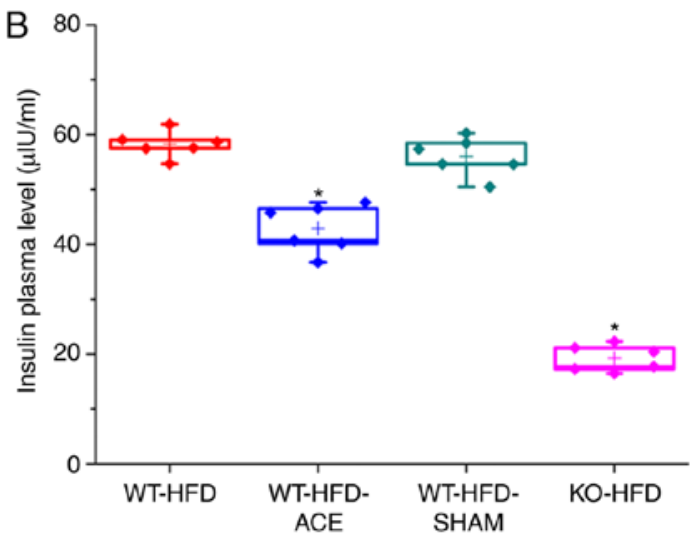

C

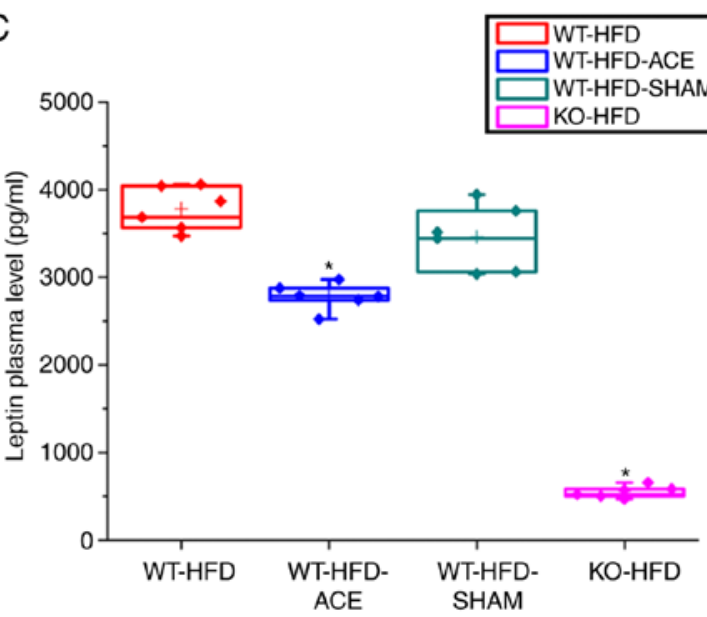

Figure 3. ELISA results for fasting glucose insulin and leptin plasma level. A graphs present the comparisons in fasting plasma levels of (A) glucose (B) insulin and (C) leptin in the WT-HFD, WT-HFD-ACE, WT-HFD-SHAM and KO-HFD groups. There were significant increases in fasting glucose plasma levels in the WT-HFD and WT-HFD-SHAM groups. ${ }^{*} \mathrm{P}<0.05$. Conversely, significantly decreased plasma levels of glucose, insulin, and leptin were observed in the WT-HFD-ACE and KO-HFD groups, which also demonstrated decreased body weight. "P<0.05. WT, wild-type; ND, normal diet; HFD, high-fat diet; ACE, acupoint catgut embedding; KO, knockout.

in the WT-HFD groups (Fig. 4E and F). There was a significant increase in $\mathrm{p}-\mathrm{PKC} \varepsilon$ protein expression in the WT-HFD-SHAM group compared with that in the WT-HFD-ACE group. In the nucleus, there was a correspondingly significant difference of $\mathrm{p}-\mathrm{NF}-\kappa \mathrm{B}$ protein levels between the obese groups; specifically, there were increased levels of $\mathrm{p}-\mathrm{NF}-\kappa \mathrm{B}$ in the WT-HFD and WT-HFD-SHAM groups compared with those in the WT-HFD-ACE and KO-HFD groups. In addition, p-CREB expression exhibited a similar pattern to that observed in the protein expression levels of the WT-HFD, WT-HFD-ACE, WT-HFD-SHAM and KO-HFD groups (Fig. 4K).

Effect of ACE treatment on proteins of TRPV1 and associated molecules in the PFC. The expression of the proteins in the PFC was examined to determine the association between obesity and corresponding psychological problems, such as depression, mediated through TRPV1-associated signaling molecules. There was a significant increase in TRPV1 expression levels in the WT-HFD-ACE treatment group compared with that in the WT-HFD and WT-HFD-SHAM treatment groups. The KO-HFD group exhibited a significant decrease in the protein level compared with that in the other three groups. The data revealed contrasting results between the hypothalamus and PFC. It was identified that the p-PI3K, p-Akt and p-mTOR expression levels were significantly decreased in the WT-HFD and WT-HFD-SHAM treatment group but were significantly increased in the WT-HFD-ACE and KO-HFD groups. Additionally, the expression levels of p-ERK, p-p38 and p-JNK exhibited similar trends to other proteins. The levels of p-ERK, p-p38 and p-JNK were significantly increased in the WT-HFD-ACE and KO-HFD groups. This increase was abolished in the WT-HFD and WT-HFD-SHAM groups when compared with the other two groups, WT-HFD-ACE and KO-HFD. There was a statistical decrease in the expression of p-PKC $\varepsilon$ and p-PKAII $\alpha$ between the WT-HFD and WT-HFD-SHAM groups (Fig. 5F). However, much larger significant increases in the WT-HFD-ACE and $\mathrm{KO}-\mathrm{HFD}$ groups were observed. Lastly, the levels of $\mathrm{p}-\mathrm{NF}-\kappa \mathrm{B}$ and $\mathrm{p}$-CREB were investigated, and a significant variation between the obese groups and groups with normal body weight was observed. Increased values were observed in the WT-HFD-ACE and KO-HFD groups, whereas decreased values were identified in the WT-HFD and WT-HFD-SHAM groups.

Effect of weight control on TRPVI and associated molecules in the hypothalamus and NTS. Immunofluorescence was used to measure the expression of TRPV1-associated downstream proteins in the hypothalamus and NTS, which is the center that regulates appetite and satiety in the brain. There was a 

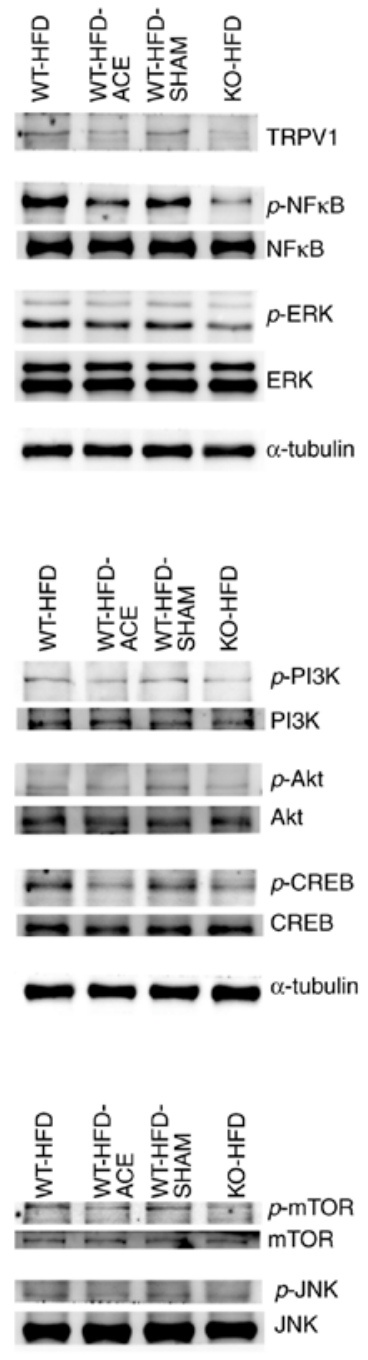

$\longrightarrow \longrightarrow-p-p 38$
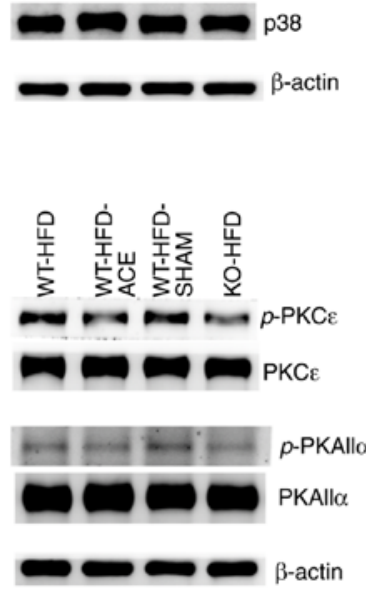
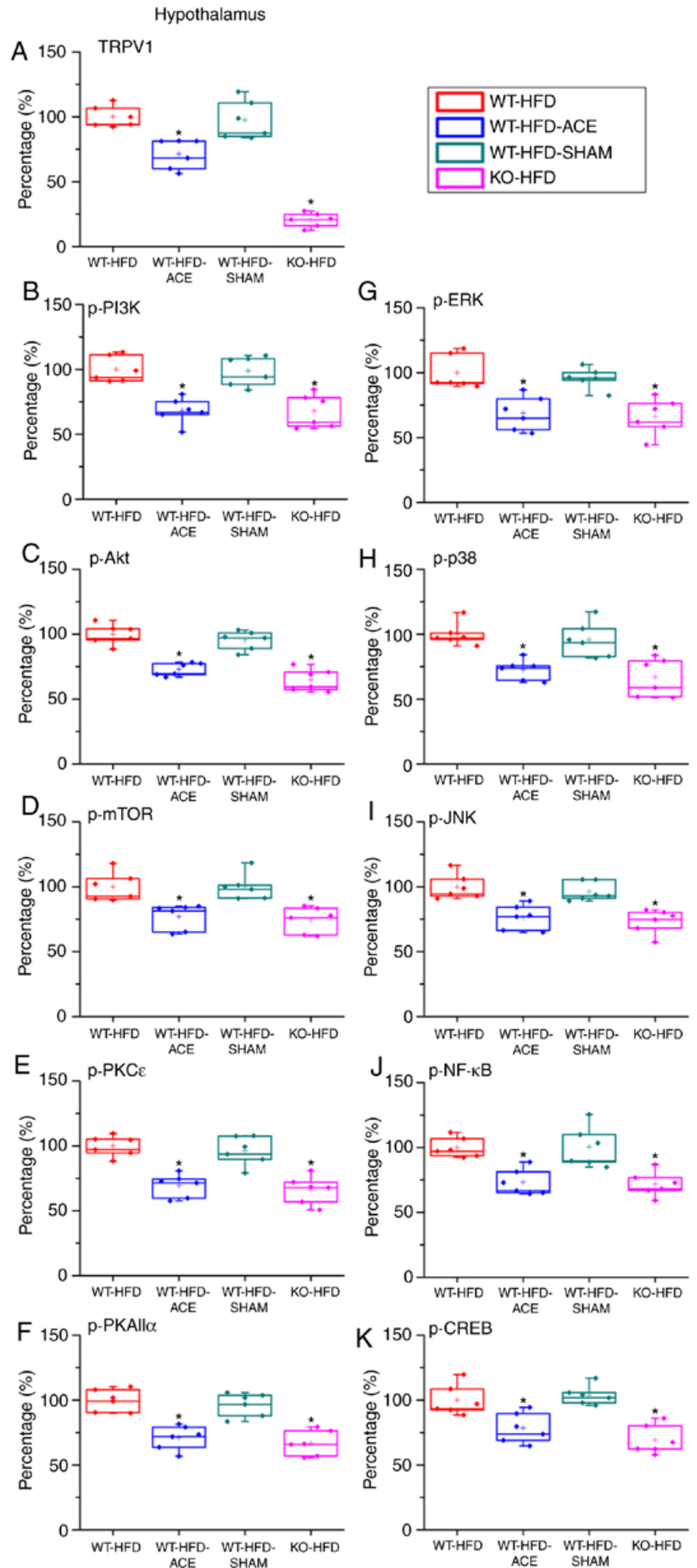

Figure 4. Expression levels of TRPV1 and associated molecules in the hypothalamus. The expression pattern of TRPV1 protein was detected in the following groups: WT-HFD; WT-HFD-ACE; WT-HFD-SHAM; and KO-HFD. The results revealed significant increases in (A) TRPV1, (B) p-PI3K, (C) p-Akt, (D) p-mTOR,

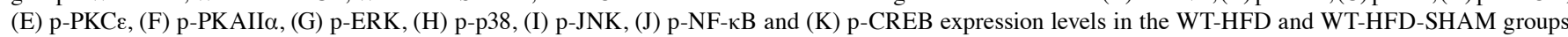
compared with the other groups ( $(\mathrm{P}<0.05)$. These increases were statistically decreased in the WT-HFD-ACE group that received ACE treatment and in the KO-HFD group that lacked the TRPV1 receptor. ${ }^{*} \mathrm{P}<0.05$. TRPV1, transient receptor vanilloid member 1; WT, wild-type; ND, normal diet; HFD, high-fat diet; ACE, acupoint catgut embedding; KO, knockout; p, phosphorylated; PKC $\varepsilon$, protein kinase C epsilon type; PKAII $\alpha$, protein kinase AII $\alpha$; ERK, extracellular signal-regulated kinase; p38, p38 mitogen-activated protein kinase; JNK, c-Jun N-terminal kinase; CREB, cyclic AMP-response element binding protein.

significant increase in TRPV1 and p-PKAII $\alpha$ immunoreactivity within the hypothalamic area of the WT-HFD and
WT-HFD-SHAM groups. However, these protein levels significantly decreased in the WT-HFD-ACE and KO-HFD groups 

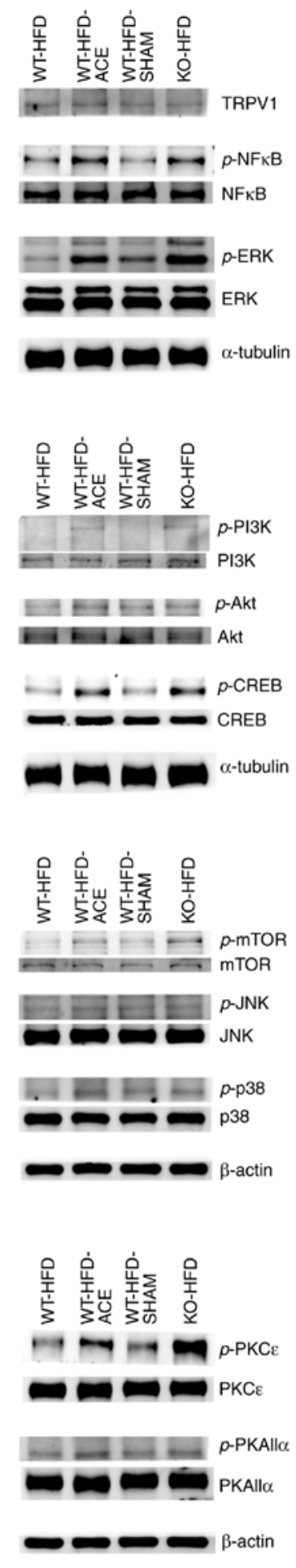

Prefrontal cortex
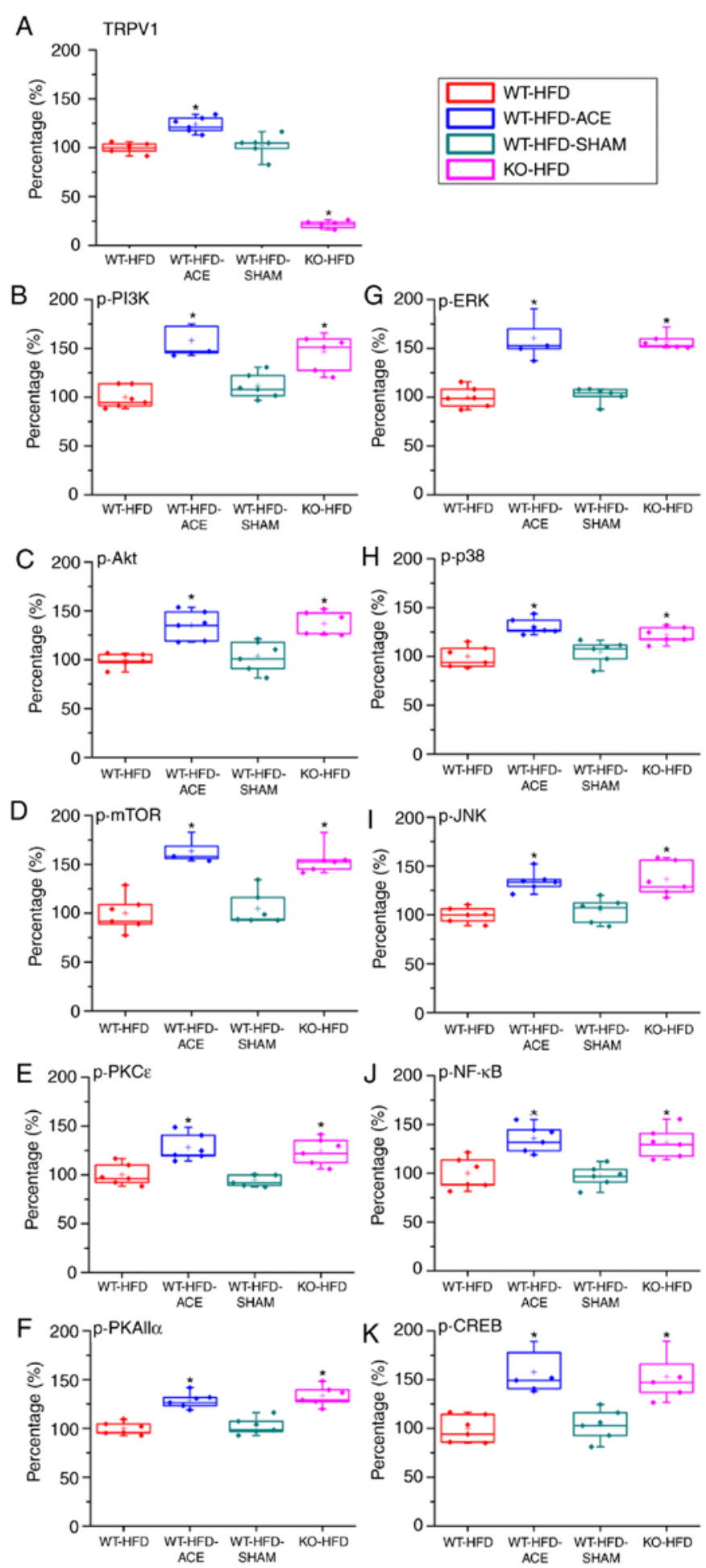

Figure 5. Expression levels of TRPV1 and associated molecules in the PFC. The expression pattern of TRPV1 protein was detected in the following groups: WT-HFD; WT-HFD-ACE; WT-HFD-SHAM; and KO-HFD. The results demonstrated significant decreases in (A) TRPV1 expression in WT-HFD, WT-HFD-SHAM and KO-HFD groups when compared with the WT-HFD-ACE group, which demonstrated a significant increase following ACE treatment. ${ }^{*} \mathrm{P}<0.05$. The results also demonstrated significant decreases in (B) p-PI3K (C) p-Akt (D) p-mTOR (E) p-PKCe (F) p-PKAII $\alpha$ (G) p-ERK (H) p-p38 (I) p-JNK (J) p-NF- $\kappa$ B and $(\mathrm{K})$ p-CREB expression levels in the WT-HFD and WT-HFD-SHAM groups compared with the other groups. * $<0.05$. The expression levels of these proteins were statistically increased in the WT-HFD-ACE and KO-HFD groups. *P<0.05. TRPV1, transient receptor vanilloid member 1 ; WT, wild-type; ND, normal diet; HFD, high-fat diet; ACE, acupoint catgut embedding; KO, knockout; PFC, prefrontal cortex; p, phosphorylated; PKCe. protein kinase C epsilon type; PKAII $\alpha$, protein kinase AII $\alpha$; ERK, extracellular signal-regulated kinase; p38, p38 mitogen-activated protein kinase; JNK, c-Jun N-terminal kinase; CREB, cyclic AMP-response element binding protein.

(Fig. 6A). Additionally, levels of p-PI3K and p-CREB (Fig. 6B) exhibited similar patterns of expression in these treatment groups. Decreased levels of TRPV1, p-PKAII $\alpha$, p-PI3K and p-CREB were also detected in the NTS (Fig. 7A and B). 
A
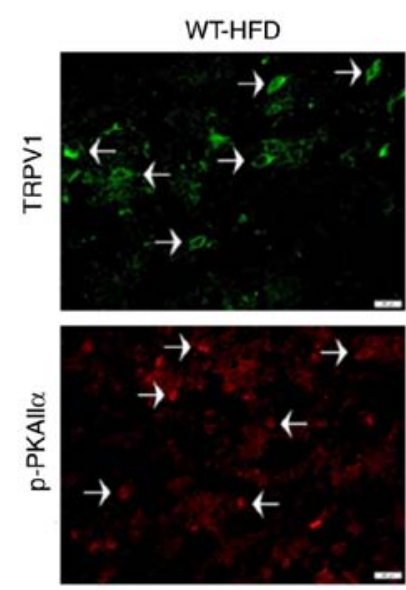

B
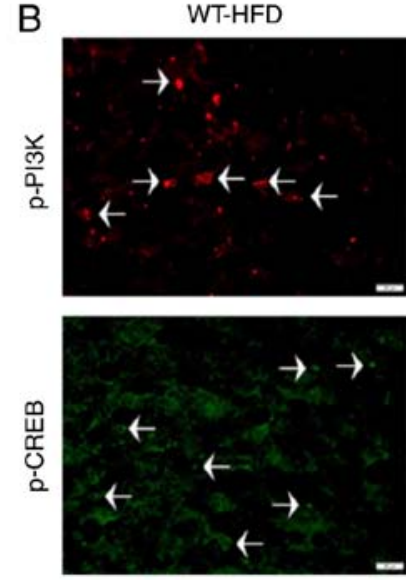

Hypothalamus
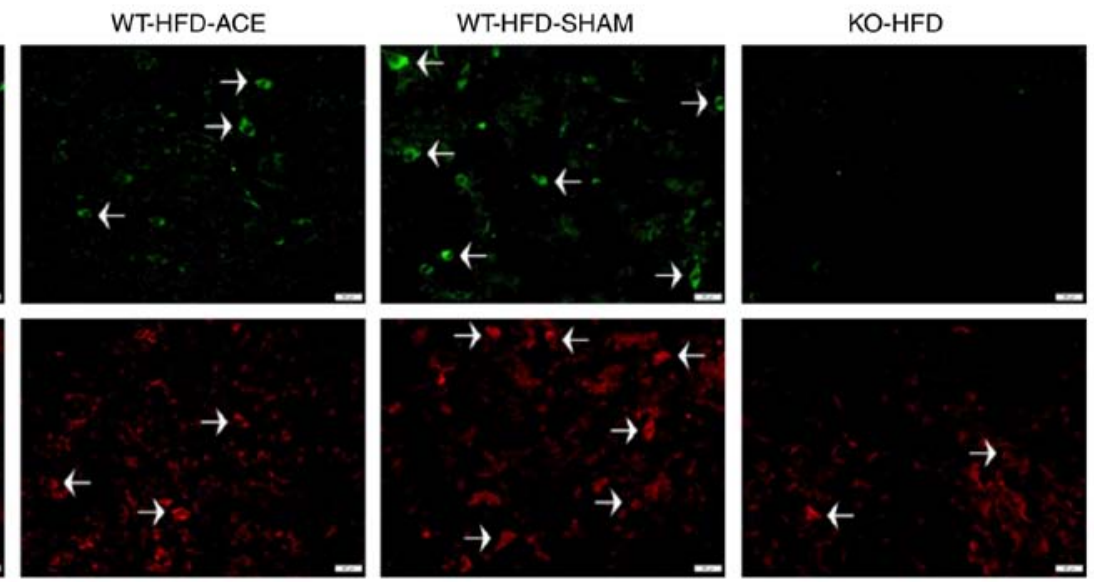

WT-HFD-ACE
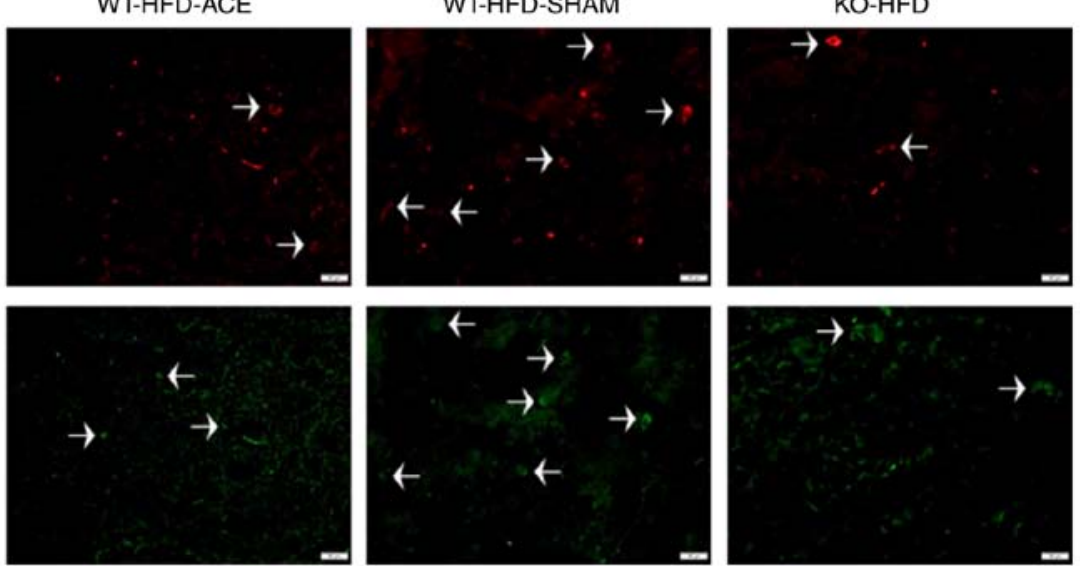

Figure 6. Expression levels of TRPV1, p-PI3K, p-CREB and p-PKAII $\alpha$ in the hypothalamus. (A) Representative immunofluorescence staining of TRPV1 (green) and p-PKAII $\alpha$ (red) and (B) representative immunofluorescence staining of p-PI3K (red) and p-CREB (green) were performed in the hypothalamus of subjects in the WT-HFD, WT-HFD-ACE, WT-HFD-SHAM and KO-HFD groups. White arrowheads indicate immunopositive cells. TRPV1, transient receptor vanilloid member 1; WT, wild-type; ND, normal diet; HFD, high-fat diet; ACE, acupoint catgut embedding; KO, knockout; p, phosphorylated; PKAII $\alpha$, protein kinase AII $\alpha$; CREB, cyclic AMP-response element binding protein.

\section{Discussion}

Obesity, which has a very high incidence rate, is largely attributed to sedentary lifestyles among the global population. Furthermore, high obesity rates have also been attributed to poor nutrition and decreased physical activity among individuals. Obesity can lead to various complications that not only affect public health but also create an economic burden in the form of increased healthcare costs. ACE treatment produces a lasting and potent stimulatory effect, thereby decreasing the treatment frequency when compared with other techniques of acupuncture. In clinical therapy, 14 days between each treatment is the interval commonly used in weight control (21). Therefore, in the present study, the catgut absorption ability in a mouse model was determined prior to initiation of the experimental model. In the present study, a time period of 7 days was established to be the optimal interval between treatments, as it is the shortest time in which the catgut can be completely absorbed. TRPV1, a calcium ion channel, is sensitively activated by capsaicin and heat, and it is distributed in various areas of the brain, including the hypothalamus, NTS and PFC. TRPV1 is heavily involved in regulating the
MAPK signaling pathway. Previous studies aimed to identify the involvement of the TRPV1 receptor in obesity. Capsaicin and associated substances increased the activation of BAT, amplified energy expenditures, and induced the browning of WAT $(28,29)$. Dietary capsaicin also possesses the ability to prevent adipogenesis, particularly vWAT, and also promote lipolysis (30). These results from previous studies suggest that TRPV1 promotes weight reduction by elevating energy expenditures and decreasing food consumption $(9,31)$.

In the present study, body mass, tissue weight and food consumption were examined to analyze feeding behavior. The results of the WT-HFD and WT-HFD-SHAM groups revealed similar increases in body mass and tissue weight, including increased weights for BAT, sWAT, vWAT, and in the liver, but not in the kidneys. There were significant decreases in BAT and vWAT in the WT-HFD-ACE treatment group compared with those in the WT-HFD and WT-HFD-SHAM groups. There was a significant decrease in food consumption in the WT-HFD-ACE group during the last week of treatment when compared with that in the WT-HFD-fed groups that did not undergo catgut insertion. Furthermore, the results revealed that the KO-ND and KO-HFD groups exhibited significant 
A
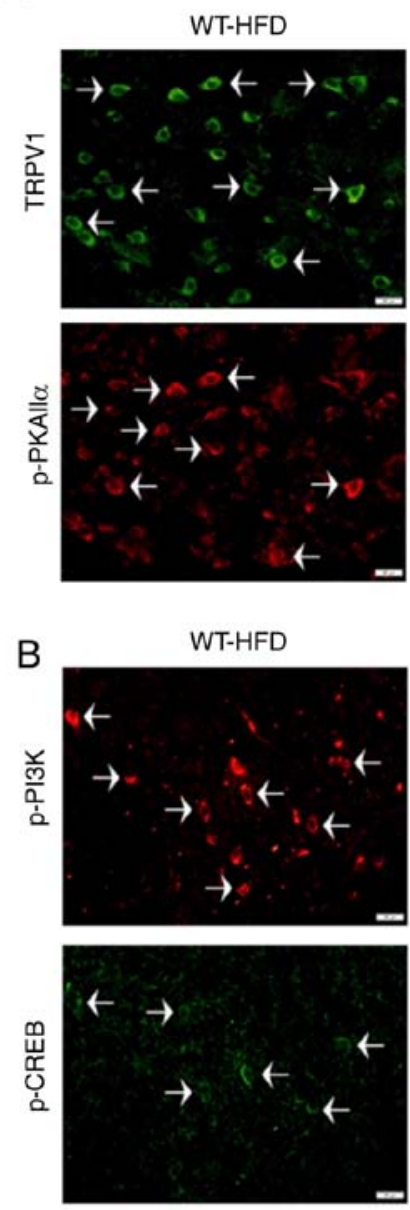

Nucleus tractus solitary
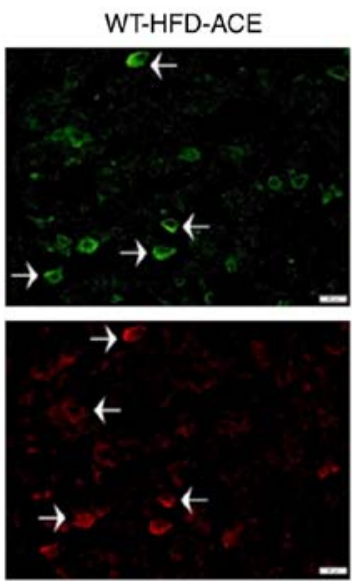

WT-HFD-ACE
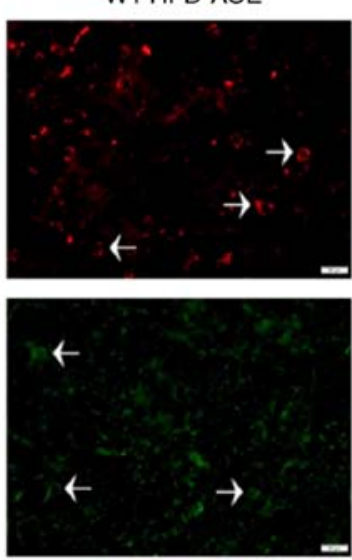

WT-HFD-SHAM

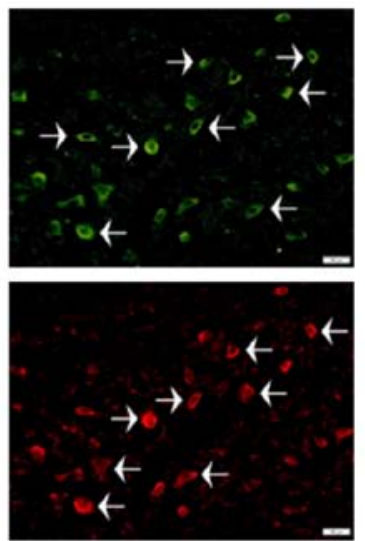

WT-HFD-SHAM
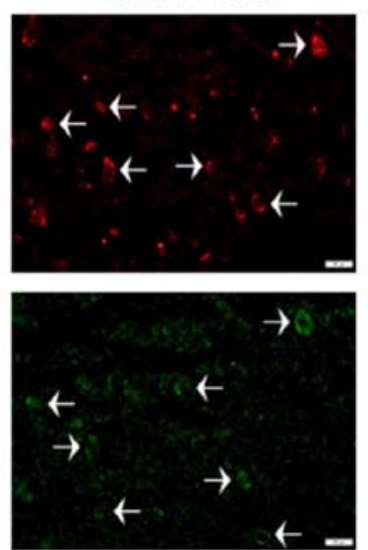

KO-HFD

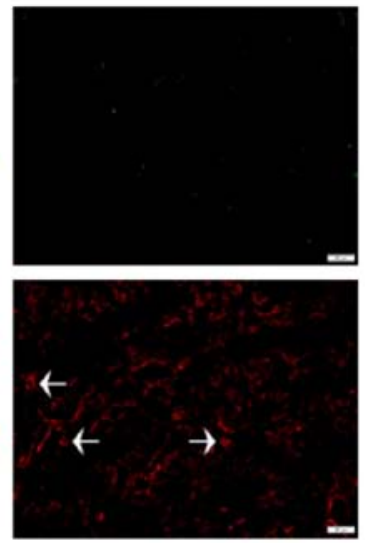

KO-HFD
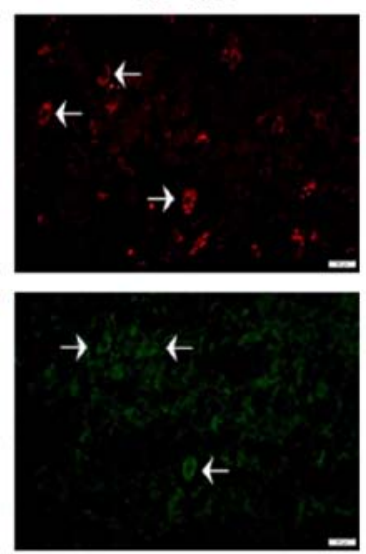

Figure 7. Expression levels of TRPV1, p-PI3K, p-CREB and p-PKAII $\alpha$ in the NTS. (A) Representative immunofluorescence staining of TRPV1 (green) and p-PKAII $\alpha$ (red) and (B) representative immunofluorescence staining of p-PI3K (red) and p-CREB (green) were performed in the NTS of subjects in the WT-HFD, WT-HFD-ACE, WT-HFD-SHAM and KO-HFD groups. White arrowheads indicate immunopositive cells. TRPV1, transient receptor vanilloid member 1; WT, wild-type; ND, normal diet; HFD, high-fat diet; ACE, acupoint catgut embedding; KO, knockout; p, phosphorylated; PKAII $\alpha$, protein kinase AII $\alpha$; CREB, cyclic AMP-response element binding protein; NTS, nucleus tractus solitarii.

decreases in weight when compared with those in the other four WT groups. Even though the KO groups did not receive any medication, the results from these groups were similar to those of the WT-HFD-ACE group. These results are supported by the results of previous studies suggesting that TRPV1 deletion protects against obesity-inducing diets (32-35). Marshall et al (36) demonstrated that although there was similarity between the body weights of WT and KO groups on an HFD, KO mice were protected against obesity-inducing hypertension, low-grade inflammation and glucose tolerance. Other previous studies described similar results in terms of body weight for WT and KO mice fed an HFD $(8,37)$. These results indicated that the KO-HFD-fed mice gained significantly more body mass compared with that in the HFD-fed WT mice (38). Accordingly, the western blot analysis results of the present study demonstrated similarities in the protein expression intensities in the hypothalamus and NTS, with greater protein density in the WT-HFD and WT-HFD SHAM treatment groups and significantly decreased density in the WT-HFD-ACE and KO-HFD groups. These results revealed the role of TRPV1 signaling in both the hypothalamus and the NTS on obesity. The fasting levels of blood, glucose, insulin and leptin revealed a proportionately correlated interaction with body mass. These data corroborate previous data, suggesting a close association between TRPV1 and glucose tolerance during obesity (36). Previous studies have identified the role of TRPV1 antagonists in promoting insulin secretion and improving insulin resistance (39-41). Additionally, a previous study examining supplementary outcomes demonstrated that TRPV1 actively participates in the regulation of leptin signaling (42). On the basis of these conflicting results concerning the effect of TRPV1 on the regulation of obesity, the results of the present study suggested that TRPV1 expression serves an integral role in the regulation and maintenance of body weight. Given the stark differences in the data from various previous studies, the association between TRPV1 and obesity remains an interesting avenue of study.

The association between obesity and depression, an important psychological problem, has also been investigated. Previous data suggest that depression is a comorbidity of obesity, and these conditions may increase the risk of developing the other condition (43). In addition, adipose tissue accumulation, which is increased in females, serves an important role in this association $(24,40,44)$. The present study exposed the different patterns of protein expression in the PFC, which is commonly associated with depression. There were lower protein levels in 
the WT-HFD and WT-HFD-SHAM groups compared with those in the WT-HFD-ACE group, which underwent catgut insertion at the ST36 acupoints. However, in the KO-HFD group, only TRPV1 levels were significantly decreased compared with those in the other WT-HFD-fed groups. By contrast, the protein expression of the MAPK molecules was significantly increased compared with that in the WT-HFD and WT-HFD-SHAM groups. Similar tendencies were exhibited among groups with decreased body weights, specifically the WT-HFD-ACE and KO-HFD groups, which demonstrated significantly increased expression levels of the MAPK proteins compared with those in the WT-HFD and WT-HFD-SHAM groups. This evidence suggests that depression serves an integral role in obesity, as indicated through the expression of MAPK proteins.

Accordingly, obesity affects multiple organ systems, even if there are only two causes, namely, excessive food intake and/or the absence of physical activity. Western and alternative medicines, such as acupuncture, are utilized to prevent the onset of obesity. Traditional Chinese acupuncture involves the stimulation of $q i$ in meridians, which are also referred to as channels. These theories have been described as the stimulation of energy through the autonomic and central nervous systems in terms of Western medical science. In more detail, the energy (or $q i$ ) has be described as the release of endogenous neurotransmitters between at least 2 cells (45-47). Various studies have comparatively analyzed the efficacy of acupuncture for the treatment of obesity. The results indicated that ACE, electroacupuncture and manual acupuncture treatment significantly decreased overall body weight and the body mass index with no distinguishable difference between these therapeutic outcomes. By contrast, placebo or sham methods was considerably less effective in decreasing body weight $(48,49)$. Although behavior modification is the ultimate solution for controlling weight, remedies that promote and maintain the performance of this treatment are in high demand. Generally, weight reduction requires more time in order to yield the desired results. Therefore, a fast-acting medication with less side effects can be combined with diet control, and exercise is optimal for the treatment of obesity.

In summary, the results of the present study identified significant associations between ACE treatment and obesity that involved TRPV1 receptors and their associated downstream signaling cascades. Additionally, the psychological problems that may arise from obesity are mediated through downstream MAPK signaling pathways. These changes were verified through the measurement of body mass and the mass of adipose tissue, TRPV1 protein expression, and the expression of proteins in the MAPK signaling pathway. The similar tendencies of the TRPV1 receptor and the downstream pathways were demonstrated through the significant decrease in protein levels in the hypothalamus and NTS. This decrease in protein expression would affect food consumption, whereas increased protein expression in the PFC would affect mental health. Nonetheless, the present study aimed to observe the effect of ACE treatment and protein expression changes in specific brain areas. Therefore, the glucose, insulin and leptin plasma level modification were studied using ELISA, in order to detect their general involvement among body and tissue weight, food consumption and protein expression. The present study also had limitations: The comparison of plasma levels and protein expression were only observed in HFD-fed subject groups, as the specified aim of the present study was to only observe the treatment effect without diet modification. For future studies, the comparison of diet modification should be considered and analyzed as a secondary independent variable, to investigate the treatment efficacy in more detail and corroborate the results of the present study.

Therefore, the present study concluded that levels of TRPV1, p-PI3K, p-mTOR, p-Akt, p-ERK, p-p38, p-JNK, p-PKC $\varepsilon, p-P K A I I \alpha, p-C R E B$ and $p-N F-\kappa B$ are involved with the development of obesity, and they may be regulated by ACE treatment. In addition, the deletion of the TRPVI gene decreased the risk of developing obesity regardless of food intake, as demonstrated by the comparison between ND and HFD feeding regimes. Lastly, the ACE treatment at the bilateral ST36 acupoint promotes weight control by decreasing food intake.

\section{Acknowledgements}

The authors would like to thank Bernice Lottering, Pei-Hsuan Chen and Hsin-Ping Ku (all China Medical University, Taichung, Taiwan) for their support with the present study. In addition, the authors would also like to thank Panupong Ngamwong (Chao Phya Abhaibhubejhr Hospital Foundation, Prachin Buri, Thailand), for their expertise with the image editing.

\section{Funding}

The present study was financially supported by MOST 107-2320-B-039-033.

\section{Availability of data and materials}

The data used to support the findings of this study are available from the corresponding author upon request.

\section{Authors' contributions}

CI designed the protocol, performed the experiments and analyzed and wrote the manuscript. YL and YH provided technical guidance and advice on the study. All authors read and approved the final manuscript.

\section{Ethics approval and consent to participate}

The use of these animals was approved by Institute of Animal Care and USE Committee of China Medical University (Permit no. 2016-061), Taiwan, following the Guide for the use of Laboratory Animals (National Academy Press).

\section{Patient consent for publication}

Not applicable.

\section{Competing interests}

The authors declare that they have no competing interests. 


\section{References}

1. GBD 2015 Obesity Collaborators, Afshin A, Forouzanfar MH, Reitsma MB, Sur P, Estep K, Lee A, Marczak L, Mokdad AH, Moradi-Lakeh M, et al: Health effects of overweight and obesity in 195 countries over 25 years. N Engl J Med 377: 13-27, 2017.

2. Cho SH, Lee JS, Thabane L and Lee J: Acupuncture for obesity: A systematic review and meta-analysis. Int J Obes (Lond) 33: 183-196, 2009

3. Thaler JP, Guyenet SJ, Dorfman MD, Wisse BE and Schwartz MW: Hypothalamic inflammation: Marker or mechanism of obesity pathogenesis? Diabetes 62: 2629-2634, 2013.

4. Coveleskie K, Kilpatrick LA, Gupta A, Stains J, Connolly L, Labus JS, Sanmiguel C and Mayer EA: The effect of the GLP-1 analogue Exenatide on functional connectivity within an NTS-based network in women with and without obesity. Obes Sci Pract 3: 434-445, 2017.

5. Lockie SH: Glucagon-like peptide-1 receptor in the brain: Role in neuroendocrine control of energy metabolism and treatment target for obesity. J Neuroendocrinol 25: 597-604, 2013.

6. Sobrino Crespo C, Perianes Cachero A, Puebla Jiménez L, Barrios V and Arilla Ferreiro E: Peptides and food intake. Front Endocrinol (Lausanne) 5: 58, 2014.

7. Valassi E, Scacchi M and Cavagnini F: Neuroendocrine control of food intake. Nutr Metab Cardiovasc Dis 18: 158-168, 2008.

8. Baskaran P, Krishnan V, Fettel K, Gao P, Zhu Z, Ren J and Thyagarajan B: TRPV1 activation counters diet-induced obesity through sirtuin-1 activation and PRDM-16 deacetylation in brown adipose tissue. Int J Obes (Lond) 41: 739-749, 2017.

9. Yoneshiro T, Aita S, Kawai Y, Iwanaga T and Saito M: Nonpungent capsaicin analogs (capsinoids) increase energy expenditure through the activation of brown adipose tissue in humans. Am J Clin Nutr 95: 845-850, 2012

10. Abdelhamid RE, Kovács KJ, Nunez MG and Larson AA Depressive behavior in the forced swim test can be induced by TRPV1 receptor activity and is dependent on NMDA receptors. Pharmacol Res 79: 21-27, 2014

11. Li HB, Mao RR, Zhang JC, Yang Y, Cao J and Xu L: Antistress effect of TRPV1 channel on synaptic plasticity and spatial memory. Biol Psychiatry 64: 286-292, 2008.

12. Ramírez-Barrantes R, Cordova C, Poblete H, Muñoz P, Marchant I, Wianny F and Olivero P: Perspectives of TRPV1 function on the neurogenesis and neural plasticity. Neural Plast 2016: 1568145, 2016.

13. Liu D, Zhu Z and Tepel M: The role of transient receptor potential channels in metabolic syndrome. Hypertens Res 31: 1989-1995, 2008.

14. Menigoz A and Boudes M: The expression pattern of TRPV1 in brain. J Neurosci 31: 13025-13027, 2011.

15. Zhang GR, Zhao H, Choi EM, Svestka M, Wang X, Cook RG and Geller AI: CaMKII, MAPK, and CREB are coactivated in identified neurons in a neocortical circuit required for performing visual shape discriminations. Hippocampus 22: 2276-2289, 2012.

16. Brown TE, Chirila AM, Schrank BR and Kauer JA: Loss of interneuron LTD and attenuated pyramidal cell LTP in Trpv1 and Trpv3 KO mice. Hippocampus 23: 662-671, 2013

17. Fernandes ES, Fernandes MA and Keeble JE: The functions of TRPA1 and TRPV1: Moving away from sensory nerves. Br J Pharmacol 166: 510-521, 2012 .

18. Nam MH, Lee SW, Na HY, Yoo JH, Paik SH, Ahn KS, Ahn YM, Ahn SY, Choi SH and Lee BC: Herbal acupuncture for the treatment of obesity. J Acupunct Meridian Stud 9: 49-57, 2016

19. Yeh ML, Chu NF, Hsu MY, Hsu CC and Chung YC: Acupoint stimulation on weight reduction for obesity: A randomized sham-controlled study. West J Nur Res 37: 1517-1530, 2015.

20. Kim SY, Shin IS and Park YJ: Effect of acupuncture and intervention types on weight loss: A systematic review and meta-analysis. Obes Res 19: 1585-1596, 2018.

21. Guo T, Ren Y, Kou J, Shi J, Tianxiao S and Liang F: Acupoint catgut embedding for obesity: Systematic review and meta-analysis. Evid Based Complement Alternat Med 2015: 401914, 2015.

22. Wu X, Mo Q, He T, Zhi N, Huang Y and Yang S: Acupoint catgut embedding for the treatment of obesity in adults: A systematic review protocol. Medicine (Baltimore) 98: e14610, 2019.

23. Garcia-Vivas JM, Galaviz-Hernandez C, Fernandez-Retana J, Pedroza-Torres A, Perez-Plasencia C, Lopez-Camarillo C and Marchat LA: Transcriptomic profiling of adipose tissue in obese women in response to acupuncture catgut embedding therapy with moxibustion. J Altern Complement Med 22: 658-668, 2016
24. National Research Council (US) Committee for the Update of the Guide for the Care and Use of Laboratory Animals: Guide for the Care and Use of Laboratory Animals, 8th edition. National Academies Press (US), Washington, DC, 2011.

25. Choowanthanapakorn M, Lu KW, Yang J, Hsieh CL and Lin YW: Targeting TRPV1 for body weight control using TRPV1(-/-) mice and electroacupuncture. Sci Rep 5: 17366, 2015.

26. Ibrahim MM: Subcutaneous and visceral adipose tissue: Structural and functional differences. Obes Rev 11: 11-18, 2010.

27. Towbin H, Staehelin T and Gordon J: Electrophoretic transfer of proteins from polyacrylamide gels to nitrocellulose sheets: Procedure and some applications. Proc Natl Acad Sci USA 76: 4350-4354, 1979

28. Baskaran P, Krishnan V, Ren J and Thyagarajan B: Capsaicin induces browning of white adipose tissue and counters obesity by activating TRPV1 channel-dependent mechanisms. Br J Pharmacol 173: 2369-2389, 2016.

29. Saito M, Yoneshiro $\mathrm{T}$ and Matsushita M: Activation and recruitment of brown adipose tissue by cold exposure and food ingredients in humans. Best Pract Res Clin Endocrinol Metab 30: 537-547, 2016.

30. Zhang LL, Yan Liu D, Ma LQ, Luo ZD, Cao TB, Zhong J, Yan ZC, Wang LJ, Zhao ZG, Zhu SJ, et al: Activation of transient receptor potential vanilloid type-1 channel prevents adipogenesis and obesity. Circ Res 100: 1063-1070, 2007.

31. Wang X, Miyares RL and Ahern GP: Oleoylethanolamide excites vagal sensory neurones, induces visceral pain and reduces short-term food intake in mice via capsaicin receptor TRPV1. J Physiol 564: 541-547, 2005.

32. Cui J and Himms-Hagen J: Long-term decrease in body fat and in brown adipose tissue in capsaicin-desensitized rats. Am J Physiol 262: R568-R573, 1992.

33. Melnyk A and Himms-Hagen J: Resistance to aging-associated obesity in capsaicin-desensitized rats one year after treatment. Obes Res 3: 337-344, 1995 .

34. Motter AL and Ahern GP: TRPV1-null mice are protected from diet-induced obesity. FEBS Lett 582: 2257-2262, 2008.

35. Chen J, Li L, Li Y, Liang X, Sun Q, Yu H, Zhong J, Ni Y, Chen J, Zhao Z, et al: Activation of TRPV1 channel by dietary capsaicin improves visceral fat remodeling through connexin43-mediated $\mathrm{Ca}^{2+}$ influx. Cardiovasc Diabetol 14: 22, 2015.

36. Marshall NJ, Liang L, Bodkin J, Dessapt-Baradez C, Nandi M, Collot-Teixeira S, Smillie SJ, Lalgi K, Fernandes ES, Gnudi L and Brain SD: A role for TRPV1 in influencing the onset of cardiovascular disease in obesity. Hypertension 61: 246-252, 2013.

37. Kentish SJ, Frisby CL, Kritas S, Li H, Hatzinikolas G, O'Donnell TA, Wittert GA and Page AJ: TRPV1 channels and gastric vagal afferent signalling in lean and high fat diet induced obese mice. PLoS One 10: e0135892, 2015.

38. Lee E, Jung DY, Kim JH, Patel PR, Hu X, Lee Y, Azuma Y, Wang HF, Tsitsilianos N, Shafiq U, et al: Transient receptor potential vanilloid type-1 channel regulates diet-induced obesity, insulin resistance, and leptin resistance. FASEB J 29: 3182-3192, 2015.

39. Tanaka H, Shimaya A, Kiso T, Kuramochi T, Shimokawa T and Shibasaki M: Enhanced insulin secretion and sensitization in diabetic mice on chronic treatment with a transient receptor potential vanilloid 1 antagonist. Life Sci 88: 559-563, 2011.

40. Zhang S, Ma X, Zhang L, Sun H and Liu X: Capsaicin reduces blood glucose by increasing insulin levels and glycogen content better than capsiate in streptozotocin-induced diabetic rats. J Agric Food Chem 65: 2323-2330, 2017.

41. Zsombok A and Derbenev AV: TRP channels as therapeutic targets in diabetes and obesity. Pharmaceuticals (Basel) 9: E50, 2016.

42. Zsombok A, Jiang Y, Gao H, Anwar IJ, Rezai-Zadeh K, Enix CL, Münzberg H and Derbenev AV: Regulation of leptin receptor-expressing neurons in the brainstem by TRPV1. Physiol Rep 2: e12160, 2014

43. Pratt LA and Brody DJ: Depression and obesity in the U.S. adult household population, 2005-2010. NCHS Data Brief 1-8, 2014

44. Derry HM, Padin AC, Kuo JL, Hughes S and Kiecolt-Glaser JK: Sex differences in depression: Does inflammation play a role? Curr Psychiatry Rep 17: 78, 2015.

45. Biella G, Sotgiu ML, Pellegata G, Paulesu E, Castiglioni I and Fazio F: Acupuncture produces central activations in pain regions. Neuroimage 14: 60-66, 2001. 
46. Omura Y: Connections found between each meridian (heart, stomach, triple burner, etc.) \& organ representation area of corresponding internal organs in each side of the cerebral cortex; release of common neurotransmitters and hormones unique to each meridian and corresponding acupuncture point \& internal organ after acupuncture, electrical stimulation, mechanical stimulation (including Shiatsu), soft laser stimulation or Qi Gong. Acupunct Electrother Res 14: 155-186, 1989.

47. Zhang ZJ, Wang XM and McAlonan GM: Neural acupuncture unit: A new concept for interpreting effects and mechanisms of acupuncture. Evid Based Complement Alternat Med 2012: $429412,2012$.
48. Sheng J, Jin X, Zhu J, Chen Y and Liu X: The effectiveness of acupoint catgut embedding therapy for abdominal obesity: A systematic review and meta-analysis. Evid Based Complement Alternat Med 2019: 9714313, 2019.

49. Zhang Y, Li J, Mo G, Liu J, Yang H, Chen X, Liu H, Cai T, Zhang X, Tian X, et al: Acupuncture and related therapies for obesity: A network meta-analysis. Evid Based Complement Alternat Med 2018: 9569685, 2018.

(i) () $($ This work is licensed under a Creative Commons cc) Attribution-NonCommercial-NoDerivatives 4.0 International (CC BY-NC-ND 4.0) License. 